\title{
Prevalence and Management Challenges in Central Post-Stroke Neuropathic Pain: A Systematic Review and Meta-analysis
}

\author{
Andreas Liampas · Nikolaos Velidakis • Tiffany Georgiou • \\ Athina Vadalouca - Giustino Varrassi - Georgios M. Hadjigeorgiou • \\ Georgios Tsivgoulis · Panagiotis Zis (D
}

Received: April 15, 2020 / Published online: May 23, 2020

(C) The Author(s) 2020

\begin{abstract}
Introduction: Central post-stroke pain (CPSP) is defined as the neuropathic pain that arises either acutely or in the chronic phase of a cerebrovascular event and is a result of central lesions of the somatosensory tract. The aim of this systematic review and meta-analysis was to
\end{abstract}

Digital Features To view digital features for this article go to https://doi.org/10.6084/m9.figshare.12287795.

Electronic supplementary material The online version of this article (https://doi.org/10.1007/s12325020-01388-w) contains supplementary material, which is available to authorized users.

\section{A. Liampas}

Academic Directorate of Neurosciences, Sheffield

Teaching Hospitals NHS Foundation Trust,

Sheffield, UK

N. Velidakis · T. Georgiou · G. M. Hadjigeorgiou .

P. Zis $(\bowtie)$

Medical School, University of Cyprus, Nicosia,

Cyprus

e-mail: takiszis@gmail.com

A. Vadalouca

Pain and Palliative Care Center, Athens Medical

Center, Athens, Greece

G. Varrassi

Paolo Procacci Foundation, Roma, Italy

G. Tsivgoulis

Second Department of Neurology, "Attikon"

University Hospital, National and Kapodistrian

University of Athens, Athens, Greece establish the prevalence of CPSP, to describe its characteristics, and to discuss the associated management challenges.

Methods: After a systematic Medline search, we identified 69 papers eligible to be included.

Results: The pooled prevalence of CPSP in patients with stroke at any location was $11 \%$ (95\% CI 7-18\%), which can increase to more than $50 \%$ in the subgroups of patients with medullary or thalamic strokes. CPSP onset coincides with stroke occurrence in $26 \%$ of patients (95\% CI 18-35\%); CPSP manifests within a month since symptom onset in $31 \%$ of patients (95\% CI 22-42\%), and occurs between the first month and the first year in $41 \%$ of patients (95\% CI 33.9-49.0\%). CPSP develops more than 12 months after stroke onset in 5\% of patients (95\% CI 3-8\%).

Conclusions: Clinicians should look for any evidence of central neuropathic pain for at least 12 months after stroke. Both pharmacological and non-pharmacological interventions can be used for the management of CPSP. Lamotrigine has the strongest evidence (Level II of evidence, derived from small randomized controlled trials) for being effective in the management of CPSP. Future research should focus on well-designed trials of pharmacological and non-pharmacological interventions aiming to relief CPSP, which is a very common but often neglected pain syndrome. 
Keywords: Central neuropathic pain; CPSP; Management; Stroke

\section{Key Summary Points}

Central post-stroke pain affects $11 \%$ of patients with stroke

More than $50 \%$ of patients with a strokes affecting the somatosensory tract will develop central post-stroke pain

More than 50\% of patients that will develop central post-stroke pain will do so in the first month after stroke

Clinicians should look for any evidence of central neuropathic pain for at least 12 months after stroke

\section{INTRODUCTION}

According to the International Association for the Study of Pain (IASP), neuropathic is any pain caused by a lesion or disease of the somatosensory system $[1,2]$. It can be spontaneous or evoked and may manifest as an extreme response to a painful stimulus (hyperalgesia) or a painful response to a normally painless stimulus (allodynia) [1].

Clinically, neuropathic syndromes are characterized by a combination of various symptoms other than pain, such as tingling, numbness, or pins and needles sensations. Such syndromes can be classified into two categories: those that arise from damage in the peripheral nervous system and those that are a consequence of a central lesion or condition [3].

Central post-stroke pain (CPSP) is defined as the neuropathic pain that arises either acutely or in the chronic phase of a cerebrovascular event (ischemic or hemorrhagic) and is a result of central lesions of the somatosensory tract [4].

The aim of this systematic review and metaanalysis was twofold; to establish the prevalence and describe the characteristics of CPSP using a meta-analytic approach to systematically review the literature regarding the associated management challenges.

\section{METHODS}

\section{Protocol Registration}

This review was prospectively registered to PROSPERO, an international prospective register of systematic reviews (registration number CRD42019157496).

\section{Literature Search Strategy}

A systematic literature search was performed on November 30, 2019 in the PubMed database. For the search, two Medical Subject Heading (MeSH) terms were used. Term A was "poststroke" OR "post-stroke". Term B was "neuropathic pain". No filters were applied. We also perused the reference lists of the included papers so as to try and include further papers reporting on CPSP.

\section{Inclusion Criteria}

Articles eligible to be included in this review were required to meet the following criteria:

1. Papers had to report on subjects with a definite diagnosis of CPSP.

2. Human subjects were involved.

3. The article was written in English language.

4. Papers were of adequate quality as described below.

\section{Exclusion Criteria}

Articles meeting the following criteria were excluded from our review:

1. Case series/cohorts with less than ten patients

2. With less than ten patients per treatment arm

3. Articles (i.e., review articles, letters, medical hypotheses, etc.) 
4. Studies not providing detailed clinical data regarding patients with CPSP

5. Pharmacological or non-pharmacological studies where CPSP relief was not the primary aim

6. Animal studies

7. Articles or papers from the same research teams describing the same patient population

All article abstracts were screened in triplicate in a blinded fashion. Those found not complying with the inclusion criteria were removed and any controversies were dealt with consensus during a face-to-face meeting, in which the abstracts were reviewed. All remaining papers were screened again as a full article by at least three authors and conflicts were settled as previously reported.

\section{Quality Assessment of Included Studies}

For prevalence studies, we evaluated their methodological quality using a checklist adapted from Hoy et al. [5]. It consists of nine questions that assess the representativeness of the sample, the sampling technique, the response rate, the data collection method, the measurement tools, the case definitions, and the statistical reporting. Each checked question was scored either as " 0 " or " 1 " corresponding respectively to "low risk of bias" and "high risk of bias". The total score ranged from 0 to 9 with the overall score categorised as follows: 7 to 9: "high risk of bias", 4 to 6: "moderate risk", and 0 to 3: "low risk" [6].

For management studies, we used the Cochrane Collaboration's tool for assessing the risk of bias in randomized trials [7] and the Newcastle-Ottawa scale, which is approved by the Cochrane Collaboration, for assessing the quality of non-randomized studies [8]. To determine the grading of evidence we used the classification proposed by the American Society of Interventional Pain Physicians (ASIPP) [9].

This information is available as supplementary material.

\section{Data Collection Process}

Data were extracted from each study in a structured coding scheme using Excel and included population size, gender and age distribution, prevalence data, stroke location, prognosis, clinical features of CPSP, the means of diagnosis, the means of treatment, the response to treatment, the side effects associated with the treatment, and the follow-up period of the patients, where applicable. When there was uncertainty regarding how data should be interpreted or utilized, at least three authors discussed the study in question to ensure consensus.

\section{Data Synthesis}

This study used aggregate data where possible and it is reported in accordance with the Preferred Reporting Items for Systematic Reviews and Meta-Analysis (PRISMA) guidelines [10].

\section{Statistical Analysis}

Statistical calculation of pooled proportions was conducted in R language, using the default settings of the "meta" package and the "metaprop" function with a random effects model [11]. The outcomes of interest were the proportion of patients with stroke that developed CPSP, the distribution of stroke location amongst patients with CPSP, and the timing of CPSP onset in patients who developed CPSP. The heterogeneity between studies was assessed by the $I^{2}$ test [12].

\section{Compliance with Ethics Guidelines}

This article is based on previously conducted studies and does not contain any studies with human participants or animals performed by any of the authors. 


\section{RESULTS}

\section{Study Characteristics}

The literature search produced a total of 682 results. During the eligibility assessment 615 articles were excluded. Two papers were identified through screening of the reference lists of the included papers. Ultimately, a total of 69 studies published between 1989 and 2019 were included in the present review [13-81]. The selection process is illustrated in Fig. 1 (PRISMA chart).

\section{Prevalence}

Thirty studies investigated non-selected populations of stroke sufferers of any age group for CPSP [13]. Of those, 21 evaluated patients with stroke at any location, four patients with medullary stroke, three patients with thalamic stroke, one patients with stroke affecting the somatosensory tract, and one patient with cortical stroke. Furthermore, two studies investigated non-selected populations of stroke sufferers of specific age groups (one in patients younger than 50 years old [43] and one in patients over 70 years old [44]) for CPSP.
Quality assessment of those studies showed that none of them carried a high risk of bias (Supplement 1).

Figure 2 shows the pooled prevalence of CPSP in patients with stroke at any location, following the meta-analysis of 21 available studies. The pooled prevalence was $11 \%(95 \%$ CI $7-17 \%, n=20,668$ ) [22-42]. However, there was substantial heterogeneity across the included studies $\left(I^{2}=98 \%\right)$. As seen in the respective funnel plot there was significant asymmetry indicating a probable publication bias.

Figure 3 shows the pooled prevalence of CPSP in medullary stroke using data from four studies. The pooled prevalence was 57\% (95\% CI $24-85 \%, n=216$ ) [17]. However, there was substantial heterogeneity across the included studies $\left(I^{2}=93 \%\right)$. As seen in the respective funnel plot there was significant asymmetry indicating a probable publication bias.

Figure 4 shows the pooled prevalence of CPSP in thalamic stroke using data from three studies. The pooled prevalence was 52\% (95\% CI $41-62 \%, n=93)[13,15]$. There was no heterogeneity across the included studies $\left(I^{2}=0 \%\right)$. As seen in the respective funnel plot, there was no significant asymmetry to indicate publication bias.

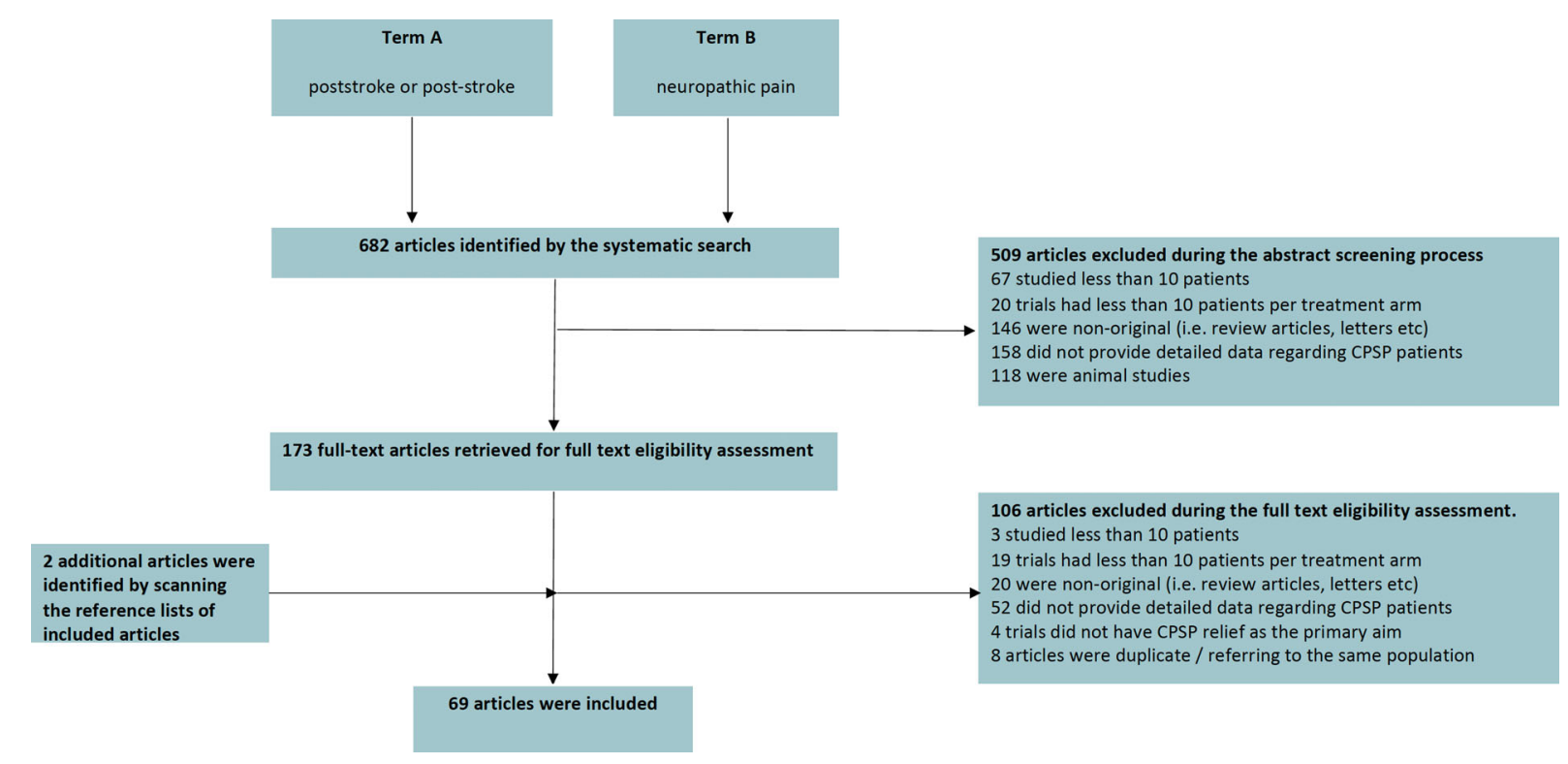

Fig. 1 PRISMA chart 
(a)

Study

Andersen 1995

Bashir 2017

Choi-Kwon 2006

Choi-Kwon 2017

Hong 2012

Jonsson 2006

Klit 2011

Klit 2014

Kong 2004

Krause 2016

Kumar 2016

O'Donnell 2013

Osama 2018

Paolucci 2016

Pellicane 2013

Raffaeli 2013

Sahin-Onat 2016

van Kollenburg 2012

Vukojevic 2018

Weimar 2002

Zeilig 2013

Logit transformed estimate

Random effects model

Heterogeneity: $I^{2}=98 \%, \tau^{2}=1.4723, p<0.01$

(b)

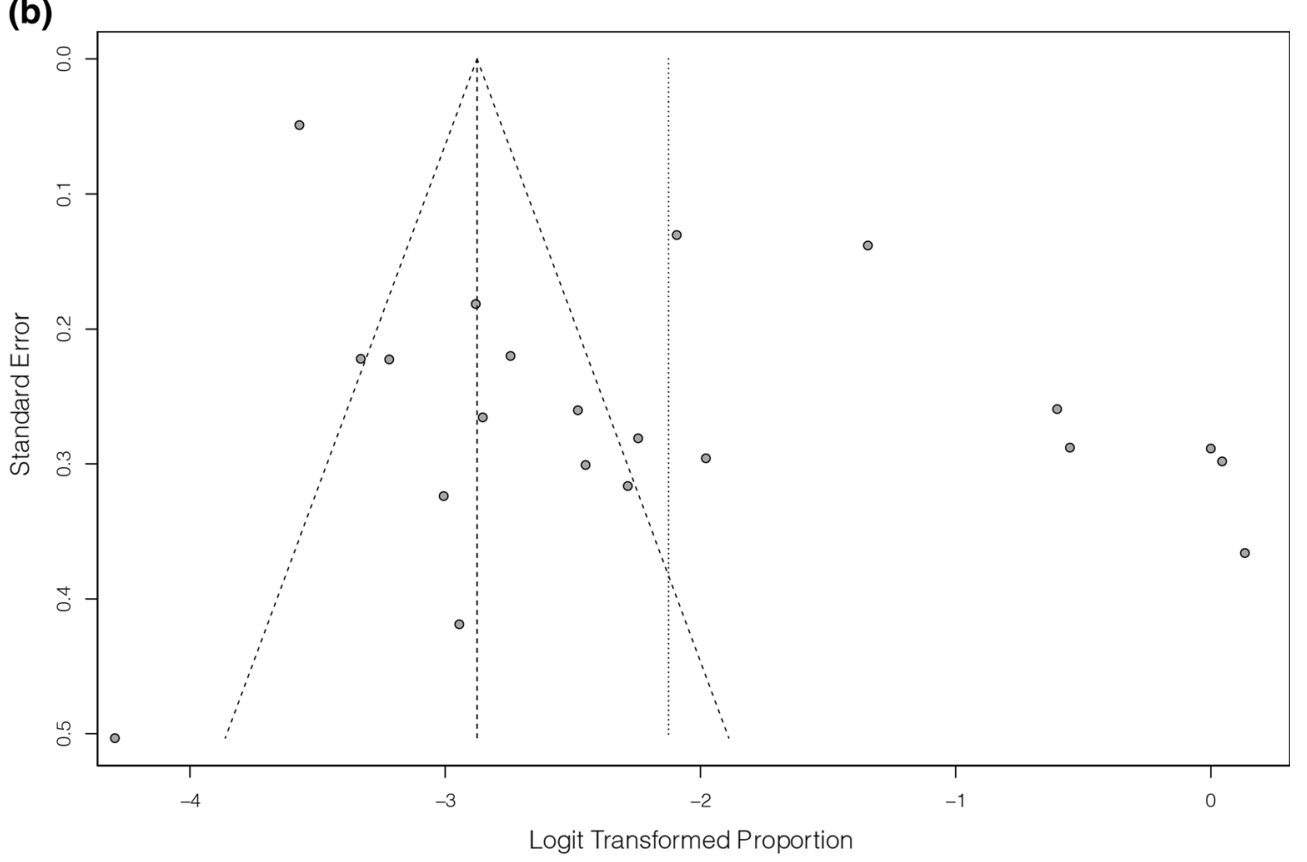

Fig. 2 Forest plot (a) for the prevalence of central post-stroke pain amongst patients with stroke at any location. The asymmetry in the respective funnel plot $(\mathbf{b})$ indicates probable publication bias

The study reporting patients that had suffered a stroke affecting the somatosensory tract, at any site, showed a similar prevalence of pain to that of patients with thalamic strokes with a CPSP of $53 \% \quad(n=30)$ [16]. The study that reported patients that had suffered a cortical stroke showed a prevalence of CPSP of $17 \%$ $(n=24)$ [21].

Both studies that investigated non-selected populations of patients with stroke of specific age groups included patients with all stroke locations. The prevalence of CPSP in the old was 

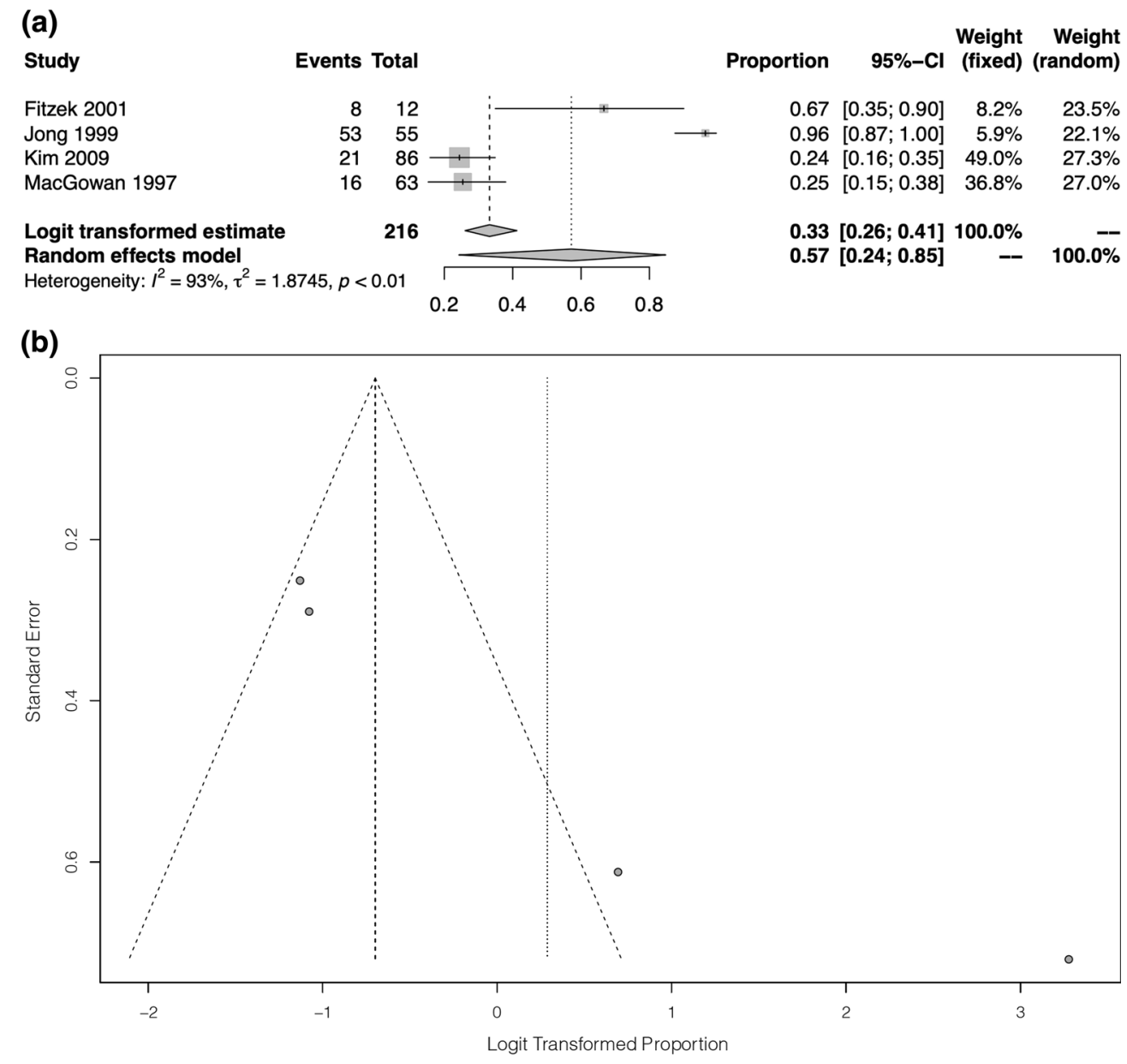

Fig. 3 Forest plot (a) for the prevalence of central post-stroke pain amongst patients with medullary stroke. The asymmetry in the respective funnel plot $(\mathbf{b})$ indicates probable publication bias

$11 \%(n=72)$ [44] and in the young $6 \%$ $(n=824)$ [43].

\section{Stroke Location in Patients with CPSP}

Twenty-three studies provided data regarding the stroke location in non-selected populations of patients with CPSP $[22,23,30,33,36,43$, 45-61]. The majority of patients with CPSP suffered a thalamic stroke $(42 \%, 95 \%$ CI $33-52 \%, I^{2}=79 \%$ ), followed by lobar strokes $\left(21 \%, 95 \%\right.$ CI $\left.14-30 \%, I^{2}=74 \%\right)$, strokes in the basal ganglia $\left(16 \%, 95 \%\right.$ CI $\left.11-23 \%, I^{2}=63 \%\right)$, brainstem strokes $\left(14 \%, 95 \% 11-17 \%, I^{2}=0 \%\right)$, and cortical strokes $(9 \%, 95 \%$ CI 5-16\%, $\left.I^{2}=72 \%\right)$. The forest plots and the respective funnel plots are available as supplementary material.

\section{Timing of CPSP}

Sixteen studies provided data regarding time of CPSP onset since stroke $[17,20,22-25,28$, $30,31,41,43,57,62-66]$. Meta-analysis of those studies showed that CPSP onset coincides with stroke occurrence in $26 \%$ of patients $(95 \%$ CI $\left.18-35 \%, I^{2}=67 \%\right)$, is within a month since stroke in $31 \%$ of patients $(95 \%$ CI $22-42 \%$, $\left.I^{2}=71 \%\right)$, is between the first month and the first year in $41 \%$ of patients (95\% CI $33-50 \%$, $I^{2}=60 \%$ ), and develops more than 12 months after the stroke in $5 \%$ of patients (95\% CI 3-8\%, $\left.I^{2}=1 \%\right)$. The forest plots and the respective 

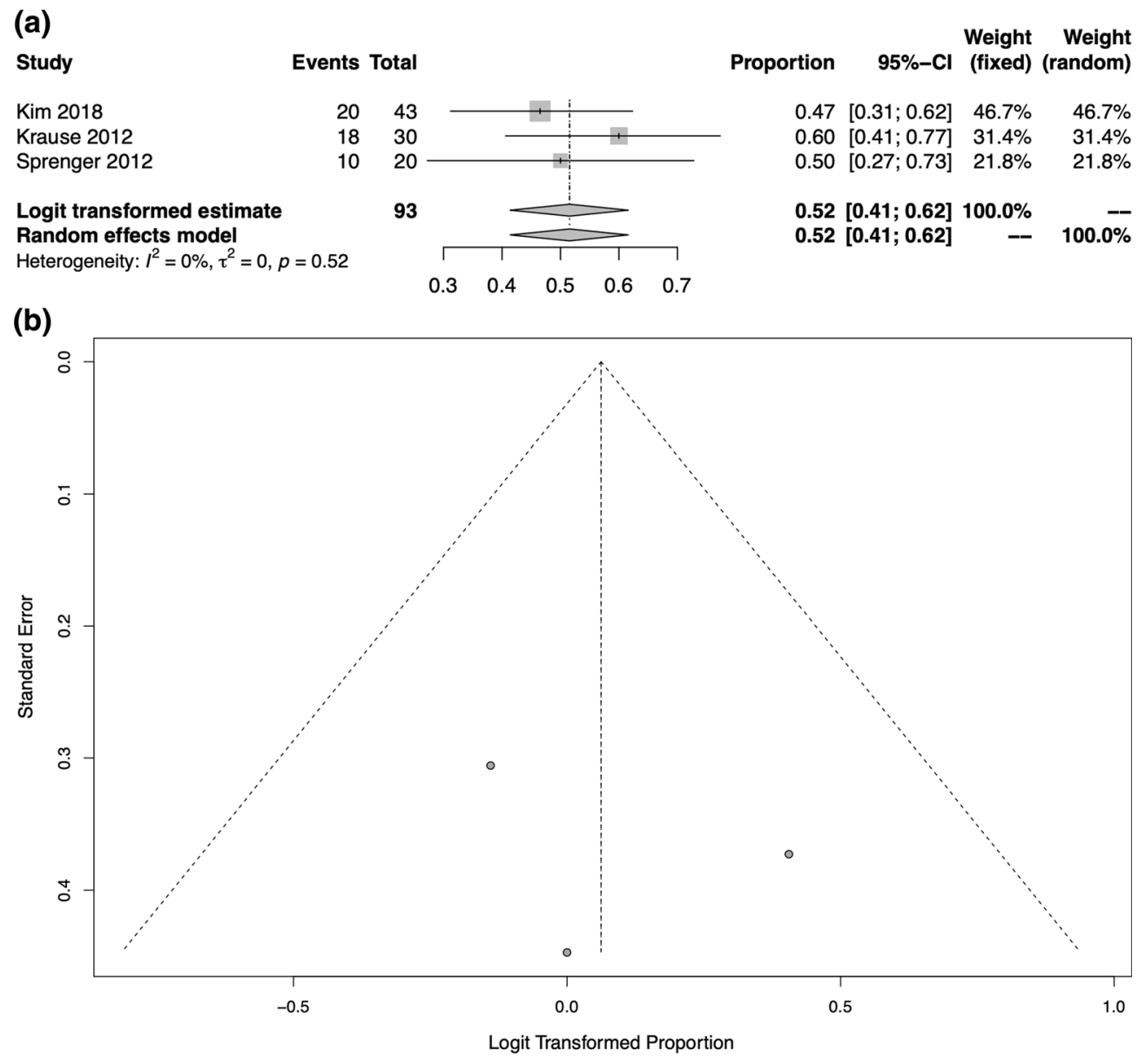

Fig. 4 Forest plot (a) for the prevalence of central post-stroke pain amongst patients with thalamic stroke. The symmetry in the respective funnel plot $(\mathbf{b})$ indicates no publication bias

funnel plots are available as supplementary material.

\section{PHARMACOLOGICAL MANAGEMENT}

\section{Selective Serotonin Reuptake Inhibitors (SSRIs)}

In an open-label, observational study, Shimodozono et al. investigated the pain relief effect of fluvoxamine in 28 patients with CPSP [66]. After 4 weeks of treatment, pain scores captured with the visual analogue scale (VAS) were significantly reduced. Interestingly, the subgroup analysis showed that the pain improvement was significant only in patients who were treated within 1 year from stroke.

\section{Tricyclic Antidepressants (TCAs)}

In a double-blind, three-phase, cross-over, placebo-controlled trial of the pain-relieving effect of amitriptyline and carbamazepine in patients with CPSP $(n=15)$ but not signs of depression, Leijon and Boivie showed that only amitriptyline produced a statistically significant reduction of pain when compared to placebo (Level III of evidence) [67].

Macgowan et al. demonstrated the response to treatment with amitriptyline in 16 patients with CPSP because of lateral medullary infarction [18]. Pain resolution was obtained in two 
participants, while all the others reported a significant reduction of pain at a tolerable level.

\section{Anticonvulsants}

Pregabalin is the most tested agent for the management of CPSP, but the evidence for its effectiveness is somewhat contradictory. Few open-label studies have reported that pregabalin is effective in the management of CPSP either as a single treatment [25] or as an add-on [68]. In an open-label randomized controlled trial (RCT), Kalita et al. compared pregabalin to lamotrigine in 30 patients with CPSP [63] and found that both were equally effective in CPSP. However, the effectiveness of pregabalin in reducing pain levels has not yet been confirmed in a placebo-controlled RCT (Level III of evidence). Kim et al. evaluated the safety, efficacy, and tolerability of pregabalin in 219 patients with CPSP [69]. Although pain reductions at endpoint did not differ significantly between pregabalin and placebo, improvements in secondary outcomes such as sleep and anxiety suggested some utility of pregabalin in the management of CPSP.

Lamotrigine has shown potential for the management of CPSP, but the current evidence is based only on small studies (Level II of evidence). Apart from the study conducted by Kalita et al. mentioned above [63], Vestergaard et al. investigated the effectiveness of orally administered lamotrigine $200 \mathrm{mg}$ daily in an intent-to-treat population of 27 patients with CPSP in a placebo-controlled RCT [70]. Pain scores in the lamotrigine group were significantly reduced compared to placebo. In the study conducted by Kalita et al., a small number of patients on lamotrigine had to discontinue treatment because of skin rash [63], whereas in the study by Vestergaard et al. lamotrigine was well tolerated with few and transient side effects [70].

In a prospective open-label observational study, Hesami et al. reported that gabapentin $300 \mathrm{mg}$ twice daily was effective in the management of CPSP in 84 patients with thalamic stroke [71].
Neither levetiracetam nor carbamazepine has been found to be effective in the management of CPSP to date $[67,72]$.

\section{Serotonin-Norepinephrine Reuptake Inhibitors (SNRIs)}

In an open-label study, Kim et al. investigated the efficacy of duloxetine as add-on in 37 patients with CPSP [73]. There was a significant reduction of pain intensity between the baseline and the follow-up assessment. Adverse events leading to withdrawal included nausea, agitation, and somnolence.

\section{Naloxone}

In a placebo-controlled RCT Bainton et al. examined the role of intravenously administered naloxone in the management of CPSP. The researchers concluded that intravenously administered naloxone is of no value in alleviating such pain [74].

\section{NON-PHARMACOLOGICAL MANAGEMENT}

\section{Magnetic Stimulation}

Repetitive transcranial magnetic stimulation (rTMS) is a safe and well-tolerated intervention that has been used for the treatment of many forms of neuropathic pain [82, 83]. The use of rTMS of the motor cortex for the treatment of CPSP has only been studied in small studies (fewer than 25 subjects in each study) with contradictory results. However, there was a great deal of heterogeneity of the studies as the studied populations had different characteristics including stroke location and the rTMS was in different sites while different protocols of stimulation were applied.

In 2014, de Oliveira et al. conducted a shamcontrolled RCT in 23 patients with intractable CPSP. The interim analysis showed a significant lack of efficacy of the active arm leading to early termination of the study [75]. However, two more recent sham-controlled 
RCTs had promising results. Khedr et al. showed that five daily sessions of rTMS over the motor cortex of patients with intractable CPSP can produce pain relief lasting 2 weeks [45] (Level III of evidence). Shimizu et al. showed that in patients with intractable lower limb CPSP deep rTMS can effectively be used to achieve shortterm pain relief [58] (Level III of evidence). A case-control rTMS study using neuronavigation suggested that restoration of abnormal cortical excitability might be one of the mechanisms underlying pain relief as a result of deep rTMS in intractable CPSP [54].

In an open-label study, with no control group, Kobayashi et al. reported that maintaining rTMS once a week as add-on therapy can help to relieve CPSP for at least 12 months [51]. Using neuronavigation, Hasan et al. suggested that the effect of rTMS may be mediated via circuitries that share the processing of noxious and thermal signals, such as the insula and the somatosensory and anterior cingulate cortices [53]. In an interesting study conducted by Goto et al. who used diffusion tensor fiber tracking, it was shown that apart from the corticospinal tract, the thalamocortical tract also plays a role in pain reduction by rTMS [81]. Moreover, it has also been suggested that presence of depression can affect the outcome of rTMS in patients with CPSP as the antalgic effect is more prominent in patients without depression [76].

\section{Electrical Stimulation of Brain and Spinal Cord}

Spinal cord stimulation (SCS) for the management of CPSP has been used in three open-label non-controlled studies, which suggested that SCS may provide improved long-term pain control (Level IV of evidence) [50, 56, 77].

Motor cortex stimulation (MCS) has been studied in small open-label non-controlled studies $[48,59,61,78]$ showing satisfactory pain control that was preserved for up to 2 years (Level IV of evidence). Katayama et al. concluded that responders to MCS were mostly patients with CPSP and intact corticospinal tract neurons, suggesting that the pain control afforded by MCS requires neuronal circuits that are maintained by those neurons [59]. Katayama et al. also conducted another study comparing the efficacy of SCS, MCS, and deep brain stimulation (DBS). They found that satisfactory pain control was obtained more frequently as the stimulation site was moved to higher levels (7\% by SCS, $25 \%$ by DBS, and $48 \%$ by MCS) [79]. These findings imply that abnormal processing of nociceptive information develops at the level of deafferentation and spreads to higher levels to a varying extent [79].

\section{Role of Prophylactic Treatment}

Lampl et al. randomly classified participants into two groups: those who received placebo $(n=20)$ and those who received amitriptyline $(n=19)$ as a prophylactic treatment for a year within the first day after the onset of stroke was diagnosed [80]. There were no statistically significant beneficial effects when participants were treated with TCAs to prevent the development of CPSP.

\section{DISCUSSION}

Using a meta-analytic approach, we established the prevalence of CPSP and showed that more than 1 in 10 of all patients with stroke will experience such pain. When looking into groups of patients with a stroke affecting the somatosensory tract, this proportion increases dramatically to more than 1 in 2 . The majority of the patients that will suffer CPSP will do so in the first month post-stroke. However, a small proportion of patients might suffer CPSP even 12 months after the cerebrovascular incident.

A clinical implication of this is that stroke specialists should consider CPSP as a symptom that can manifest not only in the acute and subacute phase of stroke. Our recommendation is that clinicians should look for any evidence of central neuropathic pain for at least 12 months after stroke as addressing this disabling symptom might improve patients' quality of life $[26,27,37,40]$.

Although the literature regarding the prevalence of CPSP is rich, this is not the case regarding the pharmacological or non- 
pharmacological management of CPSP. Currently there is a lack of large randomized trials of good quality. The best current evidence is related to the use of amitriptyline and lamotrigine, but still deriving from small RCTs. However, there are not enough studies to allow one to perform a meaningful meta-analysis.

On the other hand rTMS might be a promising intervention for the management of CPSP but there is great variation in the treatment protocols used (number of sessions, frequency of stimulation, site of stimulation) which highlights the need for future studies that are needed to shed light onto the potential of rTMS as a management option and through which protocol(s).

Our results should be interpreted with some caution given the limitations of our design. Firstly, there was a great deal of heterogeneity between studies used in the meta-analysis. Secondly, we have only searched for publication in PubMed and we, therefore, might have missed few more papers that are indexed only in other databases. Further, there is a significant publication bias as can be seen in the funnel plots. Finally, there were no restrictions in date of publication applied in this review. This was a deliberate decision made in order to review the full range of literature pertinent to the topic in question. However, a consequence of inclusion of older literature is heterogeneity as the clinical outcomes changed over time.

\section{ACKNOWLEDGEMENTS}

The authors thank study participants for their involvement in the study.

Funding. No funding or sponsorship was received for this study or publication of this article.

Authorship. All named authors meet the International Committee of Medical Journal Editors (ICMJE) criteria for authorship for this article, take responsibility for the integrity of the work as a whole, and have given their approval for this version to be published.
Disclosures. Andreas Liampas, Nikolaos Velidakis, Tiffany Georgiou, Athina Vadalouca, Georgios M Hadjigeorgiou, Georgios Tsivgoulis. Giustino Varrassi, and Panagiotis Zis are members of the journal's Editorial Board.

Compliance with Ethics Guidelines. This article is based on previously conducted studies and does not contain any studies with human participants or animals performed by any of the authors.

Data Availability. The datasets analyzed during the current study are available as supplementary material.

Open Access. This article is licensed under a Creative Commons Attribution-NonCommercial 4.0 International License, which permits any non-commercial use, sharing, adaptation, distribution and reproduction in any medium or format, as long as you give appropriate credit to the original author(s) and the source, provide a link to the Creative Commons licence, and indicate if changes were made. The images or other third party material in this article are included in the article's Creative Commons licence, unless indicated otherwise in a credit line to the material. If material is not included in the article's Creative Commons licence and your intended use is not permitted by statutory regulation or exceeds the permitted use, you will need to obtain permission directly from the copyright holder. To view a copy of this licence, visit http://creativecommons.org/licenses/by$\mathrm{nc} / 4.0 /$.

\section{REFERENCES}

1. Treede RD, Rief W, Barke A, et al. A classification of chronic pain for ICD-11. Pain. 2015;156(6):1003-7.

2. Jensen TS, Baron R, Haanpää $M$, et al. A new definition of neuropathic pain. Pain. 2011;152(10): 2204-5.

3. Meacham K, Shepherd A, Mohapatra DP, Haroutounian S. Neuropathic pain: central vs peripheral mechanisms. Curr Pain Headache Rep. 2017;21(6):28. 
4. Klit H, Finnerup NB, Jensen TS. Central post-stroke pain: clinical characteristics, pathophysiology, and management. Lancet Neurol. 2009;8(9):857-68.

5. Hoy D, Brooks P, Woolf A, et al. Assessing risk of bias in prevalence studies: modification of an existing tool and evidence of interrater agreement. J Clin Epidemiol. 2012;65:934-9.

6. Nguyen KA, Peer N, Mills EJ, Kengne AP. A metaanalysis of the metabolic syndrome prevalence in the global HIV-infected population. PLoS One. 2016;11(3):e0150970.

7. Higgins JP, Altman DG, Gøtzsche PC, et al. The Cochrane Collaboration's tool for assessing risk of bias in randomised trials. BMJ. 2011;18(343):d5928.

8. Wells GA, Shea B, O'Connell D, et al. The Newcastle-Ottawa Scale (NOS) for assessing the quality of nonrandomised studies in meta-analyses. 2008. Available from URL: http://www.ohri.ca/programs/ clinical_epidemiology/oxford.asp.

9. Manchikanti L, Falco FJ, Benyamin RM, Kaye AD, Boswell MV, Hirsch JA. A modified approach to grading of evidence. Pain Physician. 2014;17(3): E319-E325325.

10. Moher D, Liberati A, Tetzlaff J, Altman DG, PRISMA Group. Preferred reporting items for systematic reviews and meta-analyses: the PRISMA statement. PLoS Med. 2009;6(7):e1000097.

11. Langfelder P, Horvath S. WGCNA: an R package for weighted correlation network analysis. BMC Bioinform. 2008;9:559-559.

12. Higgins JP, Thompson SG. Quantifying heterogeneity in a meta-analysis. Stat Med. 2002;21: 1539-58.

13. Kim NY, Lee SC, An YS, Shin JC, Kim YW. Metabolic changes in central poststroke pain following thalamic intracerebral hemorrhage: an 18F-FDG PET study. Clin Nucl Med. 2018;43(3):62-6.

14. Sprenger T, Seifert CL, Valet $M$, et al. Assessing the risk of central post-stroke pain of thalamic origin by lesion mapping. Brain. 2012;135(8):2536-45.

15. Krause T, Brunecker P, Pittl S, et al. Thalamic sensory strokes with and without pain: differences in lesion patterns in the ventral posterior thalamus. J Neurol Neurosurg Psychiatry. 2012;83(8):776-84.

16. Hong JH, Bai DS, Jeong JY, et al. Injury of the spinothalamo-cortical pathway is necessary for central post-stroke pain. Eur Neurol. 2010;64(3):163-8.

17. Fitzek S, Baumgärtner U, Fitzek C, et al. Mechanisms and predictors of chronic facial pain in lateral medullary infarction. Ann Neurol. 2001;49(4): 493-500.

18. MacGowan DJ, Janal MN, Clark WC, et al. Central poststroke pain and Wallenberg's lateral medullary infarction: frequency, character, and determinants in 63 patients. Neurology. 1997;49(1):120-5.

19. Kim JS, Han YS. Medial medullary infarction: clinical, imaging, and outcome study in 86 consecutive patients. Stroke. 2009;40(10):3221-5.

20. Kim JS, Choi-Kwon S. Sensory sequelae of medullary infarction: differences between lateral and medial medullary syndrome. Stroke. 1999;30(12): 2697-703.

21. Kim JS. Patterns of sensory abnormality in cortical stroke. Evidence for a dichotomized sensory system. Neurology. 2007;68(3):174-80.

22. Osama A, Abo Hagar A, Elkholy S, Negm M, Abd ElRazek R, Orabi M. Central post-stroke pain: predictors and relationship with magnetic resonance imaging and somatosensory evoked potentials. Egypt J Neurol Psychiatr Neurosurg. 2018;54(1): 40-40.

23. Vukojevic Z, Dominovic Kovacevic A, Peric S, Grgic S, Bjelica B, Basta I. Frequency and features of the central poststroke pain. J Neurol Sci. 2018;391: 100-3.

24. Bashir AH, Abdullahi A, Abba MA, Mukhtar NB. Central poststroke pain: its profile among stroke survivors in Kano. Nigeria Behav Neurol. 2017;2017:9318597.

25. Kumar A, Bhoi SK, Kalita J, Misra UK. Central poststroke pain can occur with normal sensation. Clin J Pain. 2016;32(11):955-60.

26. Choi-Kwon S, Choi SH, Suh M, et al. Musculoskeletal and central pain at 1 year post-stroke: associated factors and impact on quality of life. Acta Neurol Scand. 2017;135(4):419-25.

27. Şahin-Onat Ş, Ünsal-Delialioğlu S, Kulaklı F, Özel S. The effects of central post-stroke pain on quality of life and depression in patients with stroke. J Phys Ther Sci. 2016;28(1):96-101.

28. Paolucci S, Iosa M, Toni D, et al. Prevalence and time course of post-stroke pain: a multicenter prospective hospital-based study. Pain Med. 2015;17(5):924-30.

29. Klit H, Hansen AP, Marcussen NS, Finnerup NB, Jensen TS. Early evoked pain or dysesthesia is a predictor of central poststroke pain. Pain. 2014;155(12):2699-706. 
30. Krause T, Asseyer S, Taskin B, et al. The cortical signature of central poststroke pain: gray matter decreases in somatosensory, insular, and prefrontal cortices. Cereb Cortex. 2014;26(1):80-8.

31. Pellicane AJ, Millis SR. Efficacy of methylprednisolone versus other pharmacologic interventions for the treatment of central post-stroke pain: a retrospective analysis. J Pain Res. 2013;6:557-63.

32. O'Donnell MJ, Diener HC, Sacco RL, et al. Chronic pain syndromes after ischemic stroke. Stroke. 2013;44(5):1238-43.

33. Zeilig G, Rivel M, Weingarden H, Gaidoukov E, Defrin R. Hemiplegic shoulder pain: evidence of a neuropathic origin. Pain. 2013;154(2):263-71.

34. van Kollenburg EG, Lavrijsen JC, Verhagen SC, Zuidema SU, Schalkwijk A, Vissers KC. Prevalence, causes, and treatment of neuropathic pain in dutch nursing home residents: a retrospective chart review. J Am Geriatr Soc. 2012;60(8):1418-25.

35. Hong JH, Choi BY, Chang $\mathrm{CH}$, et al. The prevalence of central poststroke pain according to the integrity of the spino-thalamo-cortical pathway. Eur Neurol. 2012;67(1):12-7.

36. Klit H, Finnerup NB, Andersen G, Jensen TS. Central poststroke pain: a population-based study. Pain. 2011;152(4):818-24.

37. Kong KH, Woon VC, Yang SY. Prevalence of chronic pain and its impact on health-related quality of life in stroke survivors. Arch Phys Med Rehabil. 2004;85(1):35-40.

38. Weimar C, Kloke M, Schlott M, Katsarava Z, Diener HC. Central poststroke pain in a consecutive cohort of stroke patients. Cerebrovasc Dis. 2002;14(3-4): 261-3.

39. Andersen G, Vestergaard K, Ingeman-Nielsen $M$, Jensen TS. Incidence of central post-stroke pain. Pain. 1995;61(2):187-93.

40. Choi-Kwon S, Choi JM, Kwon SU, Kang DW, Kim JS. Factors that affect the quality of life at 3 years poststroke. J Clin Neurol. 2006;2(1):34-41.

41. Raffaeli W, Minella CE, Magnani F, Sarti D. Population-based study of central post-stroke pain in Rimini district. Italy J Pain Res. 2013;6:705-11.

42. Jönsson AC, Lindgren I, Hallström B, Norrving B, Lindgren A. Prevalence and intensity of pain after stroke: a population based study focusing on patients' perspectives. J Neurol Neurosurg Psychiatry. 2006;77(5):590-5.
43. Harno H, Haapaniemi E, Putaala J, et al. Central poststroke pain in young ischemic stroke survivors in the Helsinki Young Stroke Registry. Neurology. 2014;83(13):1147-54.

44. Bowsher D. Stroke and central poststroke pain in an elderly population. J Pain. 2001;2(5):258-61.

45. Khedr EM, Kotb H, Kamel NF, Ahmed MA, Sadek R, Rothwell JC. Longlasting antalgic effects of daily sessions of repetitive transcranial magnetic stimulation in central and peripheral neuropathic pain. J Neurol Neurosurg Psychiatry. 2005;76(6):833-8.

46. Saitoh Y, Kato A, Ninomiya H, et al. Primary motor cortex stimulation within the central sulcus for treating deafferentation pain. Acta Neurochir Suppl. 2003;87:149-52.

47. Tang SC, Lee LJ, Jeng JS, et al. Pathophysiology of central poststroke pain: motor cortex disinhibition and its clinical and sensory correlates. Stroke. 2019;50(10):2851-7.

48. Zhang X, Zhu H, Tao W, Li Y, Hu Y. Motor cortex stimulation therapy for relief of central post-stroke pain: a retrospective study with neuropathic pain symptom inventory. Stereotact Funct Neurosurg. 2018;96(4):239-43.

49. Jang SH, Lee J, Yeo SS. Central post-stroke pain due to injury of the spinothalamic tract in patients with cerebral infarction: a diffusion tensor tractography imaging study. Neural Regen Res. 2017;12(12): 2021-4.

50. Yamamoto T, Watanabe $\mathrm{M}$, Obuchi $\mathrm{T}$, et al. Importance of pharmacological evaluation in the treatment of poststroke pain by spinal cord stimulation. Neuromodulation. 2016;19(7):744-51.

51. Kobayashi M, Fujimaki T, Mihara B, Ohira T. Repetitive transcranial magnetic stimulation once a week induces sustainable long-term relief of central poststroke pain. Neuromodulation. 2015;18(4): 249-54.

52. Im SH, Ha SW, Kim DR, Son BC. Long-term results of motor cortex stimulation in the treatment of chronic intractable neuropathic pain. Stereotact Funct Neurosurg. 2015;93(3):212-8.

53. Hasan M, Whiteley J, Bresnahan R, et al. Somatosensory change and pain relief induced by repetitive transcranial magnetic stimulation in patients with central poststroke pain. Neuromodulation. 2014;17(8):731-6.

54. Hosomi K, Kishima H, Oshino S, et al. Cortical excitability changes after high-frequency repetitive transcranial magnetic stimulation for central poststroke pain. Pain. 2013;154(8):1352-7. 
55. Bowsher D, Leijon G, Thuomas KA. Central poststroke pain: correlation of MRI with clinical pain characteristics and sensory abnormalities. Neurology. 1998;51(5):1352-8.

56. Aly MM, Saitoh Y, Hosomi K, Oshino S, Kishima H, Yoshimine T. Spinal cord stimulation for central poststroke pain. Oper Neurosurg. 2010;67(3): 206-12.

57. Misra UK, Kalita J, Kumar B. A study of clinical, magnetic resonance imaging, and somatosensoryevoked potential in central post-stroke pain. J Pain. 2008;9(12):1116-22.

58. Shimizu T, Hosomi K, Maruo T, et al. Efficacy of deep rTMS for neuropathic pain in the lower limb: a randomized, double-blind crossover trial of an H-coil and figure-8 coil. J Neurosurg. 2017;127(5): 1172 .

59. Katayama Y, Fukaya C, Yamamoto T. Poststroke pain control by chronic motor cortex stimulation: neurological characteristics predicting a favorable response. J Neurosurg. 1998;89(4):585.

60. Yamamoto T, Katayama Y, Hirayama T, Tsubokawa T. Pharmacological classification of central poststroke pain: comparison with the results of chronic motor cortex stimulation therapy. Pain. 1997;72(1): 5-12.

61. Nuti C, Peyron R, Garcia-Larrea L, et al. Motor cortex stimulation for refractory neuropathic pain: four year outcome and predictors of efficacy. Pain. $2005 ; 118(1-2): 43-52$.

62. Vestergaard K, Nielsen J, Andersen G, IngemanNielsen M, Arendt-Nielsen L, Jensen TS. Sensory abnormalities in consecutive, unselected patients with central post-stroke pain. Pain. 1995;61(2): 177-86.

63. Kalita J, Chandra S, Misra UK. Pregabalin and lamotrigine in central poststroke pain: a pilot study. Neurol India. 2017;65(3):506-11.

64. Widar M, Samuelsson L, Karlsson-Tivenius S, Ahlström G. Long-term pain conditions after a stroke. J Rehabil Med. 2002;34(4):165-70.

65. Leijon G, Boivie J, Johansson I. Central post-stroke pain-neurological symptoms and pain characteristics. Pain. 1989;36(1):13-25.

66. Shimodozono M, Kawahira K, Kamishita T, Ogata A, Tohgo S, Tanaka N. Reduction of central poststroke pain with the selective serotonin reuptake inhibitor fluvoxamine. Int $\mathrm{J}$ Neurosci. 2002;112(10):1173-81.
67. Leijon G, Boivie J. Central post-stroke pain-a controlled trial of amitriptyline and carbamazepine. Pain. 1989;36(1):27-36.

68. Onouchi K, Koga H, Yokoyama K, Yoshiyama T. An open-label, long-term study examining the safety and tolerability of pregabalin in Japanese patients with central neuropathic pain. J Pain Res. 2014;7: 439-47.

69. Kim JS, Bashford G, Murphy TK, Martin A, Dror V, Cheung R. Safety and efficacy of pregabalin in patients with central post-stroke pain. Pain. 2011;152(5):1018-23.

70. Vestergaard K, Andersen G, Gottrup H, Kristensen BT, Jensen TS. Lamotrigine for central poststroke pain. A randomized controlled trial. Neurology. 2001;56(2):184-90.

71. Hesami O, Gharagozli K, Beladimoghadam N, Assarzadegan F, Mansouri B, Sistanizad M. The efficacy of gabapentin in patients with central poststroke pain. Iran J Pharm Res. 2015;14(Suppl): 95-101.

72. Jungehulsing GJ, Israel H, Safar N, et al. Levetiracetam in patients with central neuropathic poststroke pain-a randomized, double-blind, placebocontrolled trial. Eur J Neurol. 2013;20(2):331-7.

73. Kim NY, Lee SC, Kim YW. Effect of duloxetine for the treatment of chronic central poststroke pain. Clin Neuropharmacol. 2019;42(3):73-6.

74. Bainton T, Fox M, Bowsher D, Wells C. A doubleblind trial of naloxone in central post-stroke pain. Pain. 1992;48(2):159-62.

75. de Oliveira RA, de Andrade DC, Mendonça M, et al. Repetitive transcranial magnetic stimulation of the left premotor/dorsolateral prefrontal cortex does not have analgesic effect on central poststroke pain. J Pain. 2014;15(12):1271-81.

76. Ohn SH, Chang WH, Park CH, et al. Neural correlates of the antinociceptive effects of repetitive transcranial magnetic stimulation on central pain after stroke. Neurorehabil Neural Repair. 2012;26(4):344-52.

77. Tanei T, Kajita Y, Takebayashi S, Aoki K, Nakahara N, Wakabayashi T. Predictive factors associated with pain relief of spinal cord stimulation for central post-stroke pain. Neurol Med Chir (Tokyo). 2019;59(6):213-21.

78. Mertens P, Nuti C, Sindou M, et al. Precentral cortex stimulation for the treatment of central neuropathic pain. Stereotact Funct Neurosurg. 1999;73(1-4):122-5. 
79. Katayama Y, Yamamoto T, Kobayashi K, Kasai M, Oshima H, Fukaya C. Motor cortex stimulation for post-stroke pain: comparison of spinal cord and thalamic stimulation. Stereotact Funct Neurosurg. 2001;77(1-4):183-6.

80. Lampl C, Yazdi K, Röper C. Amitriptyline in the prophylaxis of central poststroke pain. Preliminary results of 39 patients in a placebo-controlled, longterm study. Stroke. 2002;33(12):3030-2.

81. Goto T, Saitoh Y, Hashimoto N, et al. Diffusion tensor fiber tracking in patients with central poststroke pain; correlation with efficacy of repetitive transcranial magnetic stimulation. Pain. 2008;140(3):509-18.

82. Zis P, Shafique F, Hadjivassiliou M, et al. Safety, tolerability, and nocebo phenomena during transcranial magnetic stimulation: a systematic review and meta-analysis of placebo-controlled clinical trials. Neuromodulation. 2019;23:291-300.

83. Aamir A, Girach A, Sarrigiannis PG, et al. Repetitive magnetic stimulation for the management of peripheral neuropathic pain: a systematic review. Adv Ther. 2020;37(3):998-1012. 